\title{
Ansatz zur Entwicklung von Referenzprozessen im Bereich des Enterprise Content Managements unter dem Aspekt der Wandlungsfähigkeit
}

\author{
Sandy Eggert \\ Lehrstubl für Wirtschaftsinformatik und Electronic Government, \\ Universität Potsdam \\ August-Bebel-Str. 89, 14482 Potsdam \\ bttp:/ / wi.uni-potsdam.de
}

\section{Einleitung}

Da das heutige globale Wettbewerbsumfeld von Unternehmen oft von starker Konkurrenz und kurzen Innovationszyklen gekennzeichnet ist (Rogalski und Ovtcharova 2009, S.64; Wiendahl 2009, S. 32; Wildemann 2006, S. 2) sind Unternehmen vermehrt unterschiedlichsten Turbulenzen ausgesetzt. Wandlungsfähigkeit ist ein Ansatz, diesen Turbulenzen adäquat zu begegnen.

Das Thema Wandlungsfähigkeit gelang über die Fabrikplanung in den Fokus von Organisationen und Unternehmen. Dabei wurde festgestellt, dass vor allem große Unternehmen von ihrer IT-Infrastruktur abhängig sind und der Forderung nach Wandlungsfähigkeit nachkommen müssen (Overby et al. 2006, S. 120). Flexibilität und Wandlungsfähigkeit sind bei Fabrikplanungsprojekten wichtige Zielgrößen (Möller et al. 2005, S. 2). Unterschiedliche Forschungsprojekte haben bislang Ansätze aufgezeigt, diese Zielgrößen auf Informationssysteme zu übertragen. Wandlungsfähigkeit wurde dabei häufig als ein über Flexibilität hinausgehendes Potenzial struktureller Maßnahmen verstanden, das im Bedarfsfall aktiviert werden kann, um Anpassungen an veränderte Rahmenbedingungen vorzunehmen. Dieses Konzept soll nun auf Prozesse des Enterprise Content Managements übertragen werden.

Enterprise Content Management (ECM) zielt auf die Erschließung aller Informationen eines Unternehmens (Eggert und Gronau 2005, S. 48) und gewinnt hinsichtlich des internationalen Wettbewerbsdrucks immer stärker an Bedeutung. Im ECM werden zur Verwaltung von Dokumenten und Content IT-Systeme und Prozesse betrachtet, in denen die Informationsobjekte und Dokumente entlang ihres Lebenszyklus verarbeitet werden. Um die Wandlungsfähigkeit des ECM zu erhöhen, müssen Konzepte eingesetzt werden, mit denen die System- und Pro- 
zesswandlungsfähigkeit verbessert werden kann. Zur Erhöhung der Wandlungsfähigkeit von IT-Systemen existieren bereits Methoden (Gronau et al. 2006, S. 24f), die erfolgreich auf ECM-Systeme adaptiert werden konnten. ECM umfasst jedoch neben der systemtechnischen Seite vor allem auch Prozessaspekte. Prozesse im Rahmen des ECM sind durch eine hohe Dokumentenintensivität gekennzeichnet und greifen idealerweise auf ECM-Systeme zu. Im Gegensatz zu ECM-Systemen, die mit bereits vorhandenen und auf ECM adaptierbare Vorgehensmodelle auf Wandlungsfähigkeit untersucht und verbessert werden können, bestehen im Bereich der Prozessverbesserung unter dem Aspekt der Wandlungsfähigkeit nur weinige nicht ECM-spezifische Ansätze (Andresen et al. 2008 und Heinen et al. 2008).

Ziel des Beitrages ist es, einen methodischen Ansatz vorzustellen, der unter Anwendung von Indikatoren zur Erhöhung der Wandungsfähigkeit, wandlungsfähige Referenzprozesse entwickelt. Diese unter Verwendung der Prozesskartographie entwickelten Referenzprozesse sollen sich durch die Eigenschaft der Wandlungsfähigkeit auszeichnen und werden im Folgenden als Kartenmuster bezeichnet.

\section{Entwicklung von Kartenmustern}

Abbildung 1 visualisiert die Vorgehensweise zur Entwicklung der wandlungsfähigen Kartenmuster. Die Phasen „Bildung der Fragmente“ und „Ableitung der Bewertungsindikatoren" stellen die Grundlage zur anschließenden Ableitung von Gestaltungshinweisen für wandlungsfähige ECM-Prozesse dar.

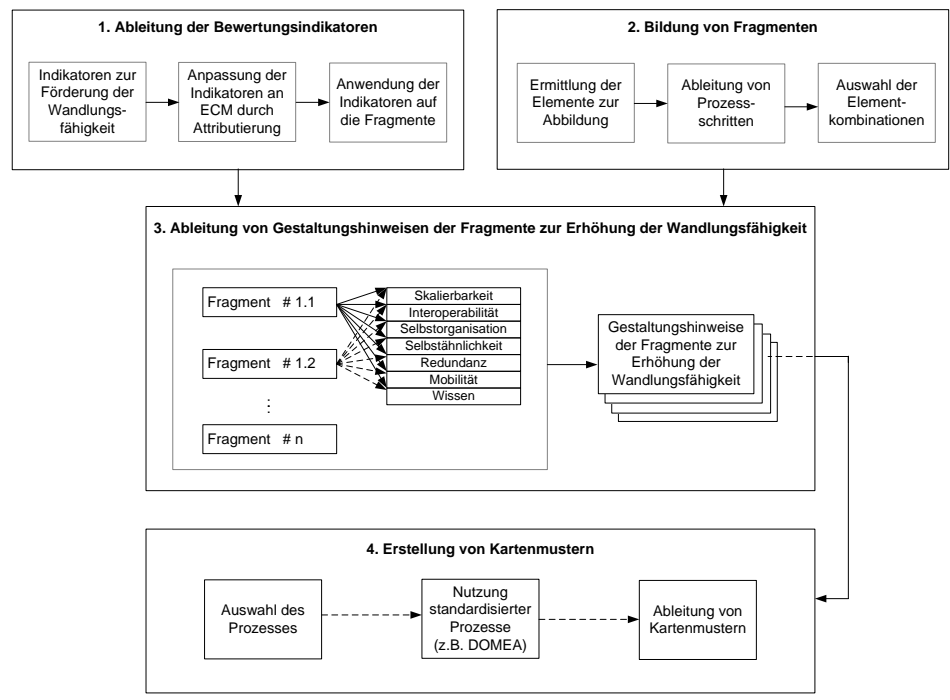

Abbildung 1: Methode zur Entwicklung wandlungsfähiger Kartenmuster 


\subsection{Ableitung der Bewertungsindikatoren für ECM-Prozesse}

Die Methode zur Entwicklung wandlungsfähiger Kartenmuster verwendet die Indikatoren zur Förderung der Wandlungsfähigkeit von Anwendungssystemen. Es besteht die Annahme, dass diese Indikatoren vor dem Hintergrund einer systemischen Betrachtungsweise (Andresen et al. 2008, S. 3) auf ECM-Prozesse angewandt werden können. Als Systembestandteile werden Prozessschritte, organisatorische Einheiten sowie Anordnungsbeziehungen der Phasen und Elemente definiert. Um die Übertragung der Indikatoren auf ECM-Prozesse durchführen zu können, werden zuvor wandlungsfähige ECM-Prozesse definiert.

\section{Tabelle 1: Attributierung der Indikatoren}

\begin{tabular}{|l|l|}
\hline Indikator & Attributierung \\
\hline Interoperabilität & $\begin{array}{l}\text { Informations- und Dokumentenaustausch, Prozessschnittstellen, Modul- und System- } \\
\text { schnittstellen, Transfer von Prozesselementen }\end{array}$ \\
\hline Modularität & Aufbau der Systeme, Aufbau und Abgrenzung der Prozessschritte, Austauschbarkeit \\
\hline Skalierbarkeit & Quantitative Anpassbarkeit, Prozesssteuerung \\
\hline Mobilität & Unabhängigkeit, örtliche und zeitliche Verfügbarkeit \\
\hline Redundanz & Effizientes Ressourcenmangt., Vermeidung unerwünschter Redundanzen \\
\hline Selbstähnlichkeit & Aufbau von ECM-Modulen, Gestaltung der Prozessschritte \\
\hline Selbstorganisation & $\begin{array}{l}\text { Prozessbasierte Kontrollparameter, Diagnosemöglichkeiten, Gestaltungsoptionen } \\
\text { (Ordnungsparameter) }\end{array}$ \\
\hline Wissen & Prozess- und Systemwissen, Anforderungsprofile, Informationsbereitstellung \\
\hline
\end{tabular}

ECM fokussiert das Management von Informationen innerhalb der Unternehmensprozesse. Die Unternehmensprozesse, die durch ein erhöhtes Dokumentenaufkommen gekennzeichnet sind, werden demnach als ECM-Prozesse bezeichnet. Ein Prozess allgemein wird basierend auf den Ergebnissen des im Forschungsprojektes IOSEW ${ }^{2}$ (Andresen et al. 2008, S. 4f) vorgestellten Betrachtungsansatz als soziotechnisches System angesehen. Dieses System kann entlang des IOSEW ${ }^{2}-$ Ansatzes genau dann als wandlungsfähig angesehen werden, wenn Änderungen des Prozessablaufs möglich sind und das System einen kontinuierlichen Prozess der Verbesserung darstellt. Ein ECM-Prozess kann basierend auf diesem Verständnis als wandlungsfähig bezeichnet werden, wenn die Kriterien der Wandlungsfähigkeit als Gestaltungsprinzipien angewandt wurden und geeignete Parameter zur Überprüfung der Performance definiert sind und kontrolliert werden können. Ein ECM-Prozess wird im Rahmen der Arbeit als wandlungsfähig bezeichnet, wenn Änderungsbedarfe durch die Elemente des Prozesses erkannt und Alternativen zur Durchführung der Änderungen von den Prozesselementen selbst generiert werden können. In Tabelle 1 wird die Übertragung der Indikatoren auf ECM-Prozesse mit dem Gestaltungsziel der Wandlungsfähigkeit verdeutlicht. Die Anpassung der Indikatoren wird mit Hilfe einer ECM-spezifischen Attributierung zum Zweck einer Operationalisierung zur Ableitung von Gestaltungsempfehlungen für ECM- 
Prozesse durchgeführt. Tabelle 2 zeigt beispielhaft die Attributierung des Indikators Interoparabilität in detaillierter Form.

Tabelle 2: Attributierung des Indikators Interoperabilität

\begin{tabular}{|l|l|}
\hline Attribut & ECM-spezifische Bedeutung \\
\hline $\begin{array}{l}\text { Informations- und } \\
\text { Dokumentenaus- } \\
\text { tausch }\end{array}$ & $\begin{array}{l}\text { Der Austausch von Informationen und Dokumenten der Prozesselemente innerhalb } \\
\text { eines Prozesses umfasst das Vorhandensein von Definitionen (z.B. Dokumentenaus- } \\
\text { tauschformate, Dokumentenformate) und die Vorgabe der Nutzung definierter } \\
\text { Medien zum Austausch (z.B. E-Mail). }\end{array}$ \\
\hline Prozessschnittstellen & $\begin{array}{l}\text { Das Attribut Prozessschnittstellen beinhaltet die Festlegung der Übergabeformate } \\
\text { zwischen einzelnen Prozessschritten, sowie Definitionen von Schnittstellen zu an- } \\
\text { grenzenden Prozessen (Vorgänger/ Nachfolger). }\end{array}$ \\
\hline $\begin{array}{l}\text { Modul- und System- } \\
\text { schnittstellen }\end{array}$ & $\begin{array}{l}\text { Modul- und Systemschnittstellen regeln die Kommunikation zwischen Systemen und } \\
\text { Systemmodulen mit Hilfe von Standards (z.B. XML, EDI). Weiterhin wird die Mög- } \\
\text { lichkeit betrachtet, einzelne Module oder Systeme miteinander verbinden zu können } \\
\text { (z.B. über Web Service). }\end{array}$ \\
\hline $\begin{array}{l}\text { Transfer von Pro- } \\
\text { zesselementen }\end{array}$ & $\begin{array}{l}\text { Der Transfer einzelner Prozesselemente ermöglicht einen einfachen Austausch bzw. } \\
\text { Übertragung von Elementen. Die Elemente des Prozesses müssen mit Hilfe einer } \\
\text { klaren Beschreibung so definiert sein, dass sie flexibel ausgetauscht oder auf andere } \\
\text { Prozesse übertragen werden können. }\end{array}$ \\
\hline
\end{tabular}

\subsection{Bildung von Fragmenten mit Gestaltungshinweisen}

Um als Referenzprozesse dienende Kartenmuster für ECM-Prozesse unter dem Aspekt der Wandlungsfähigkeit zu entwickeln, werden zuvor einzelne Prozessbausteine zur Betrachtungsgrundlage entwickelt. Ziel ist es, alle möglichen und relevanten Prozessbausteine in den Kartenmustern abzudecken, um schließlich auf Basis der Indikatoren Empfehlungen zur Verbesserung der Wandlungsfähigkeit für einen ECM-Gesamtprozess ableiten zu können. Die zu ermittelnden Prozessbausteine werden im Folgenden als Fragmente bezeichnet, da sie einzelne, spezifische Teile eines ECM-Prozesses verkörpern. Zur Bildung von Fragmenten, werden die Lebenszyklusphasen von Dokumenten und Content herangezogen. Es gilt hierbei die Annahme, dass mit Hilfe der Lebenszyklusphasen alle Prozessschritte in allen möglichen ECM-Prozessen abgebildet werden können. Da die aktuell im Bereich des ECM eingesetzten Systeme in ihrer Mehrheit eher Dokumenten- als Contentorientiert sind (Eggert und Gronau 2008, S. 443) und meist der Dokumentenverwaltung dienen (Mertins et al. 2008, S. 2), liegt der Schwerpunkt der Betrachtung zunächst auf den Dokumentenlebenszyklusphasen. Der typische Lebenszyklus eines Dokuments reicht von der Erstellung über die Nutzung und Archivierung bis hin zur Vernichtung (Kaiser et al. S.492). Jeder Lebenszyklusphase werden (siehe Beispiel in Abbildung 2) Prozesse zugeordnet, die Aktivitäten dieser Phase wiedergeben. Die dafür genutzten Prozesse sind Prozesses des ECM, die bei mehreren Praxispartnern erhoben wurden. Das mit diesem Vorgehen verbundene Ziel ist die Ermittlung der Prozessschritte, die im Fragment abgebildet werden. Das Beispiel in Abbildung 2 zeigt die Ermittlung der Prozessschritte für das Fragment 1.1, welches die Lebenszyklusphase „Erstellen“ abbildet. 


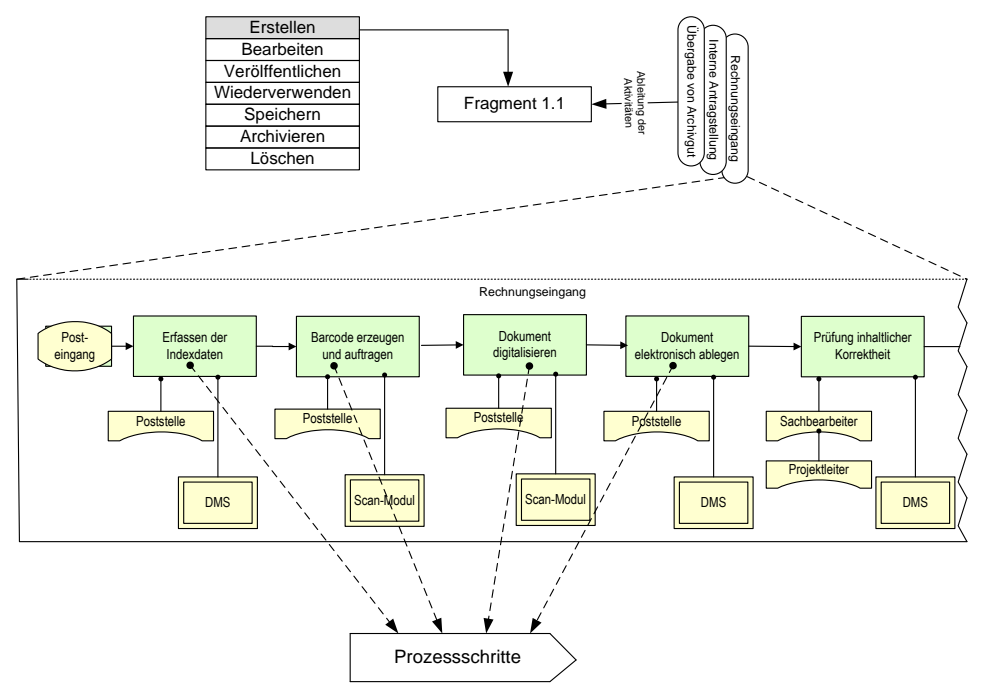

Abbildung 2: Ableitung der Prozessschritte am Beispiel von Fragment 1.1

Nach der Filterung der für die Lebenszyklusphase relevanten Prozessschritte können diese Prozessschritte zur Abbildung in den Fragmenten aufgenommen werden. Die weiteren Visualisierungselemente (z.B. System, Verbindungen) zur Darstellung in den Fragmenten können nun anhand der zugeordneten Prozesse ergänzt werden. Das Fragment „1.1: Digitalisierung“ beschreibt das Digitalisieren eines ursprünglich papierbasierten Dokuments als vorbereitende Maßnahme zur Ablage in einem IT-System.

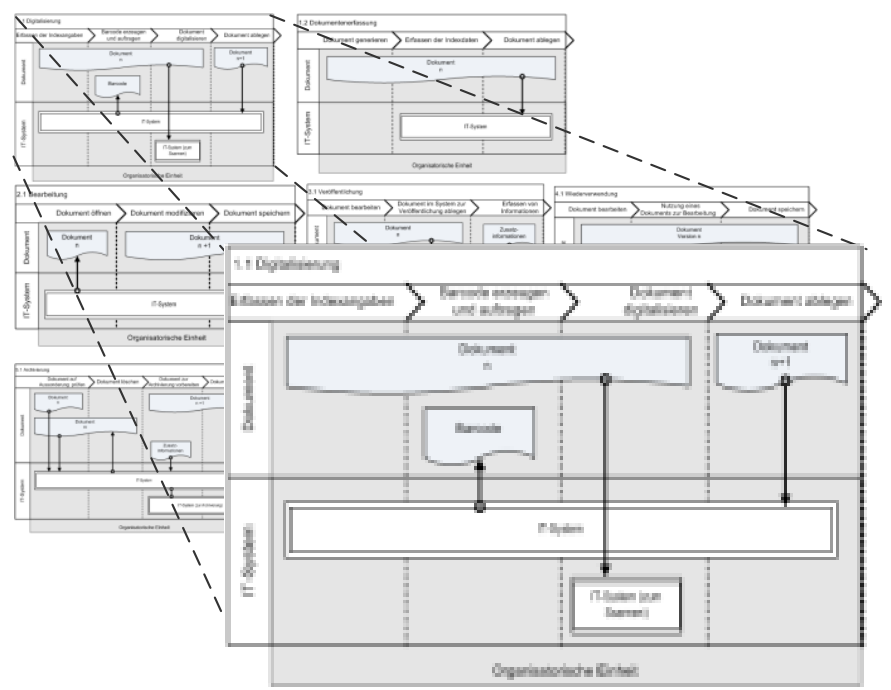

Abbildung 3: Fragment Digitalisierung 
Typische Einsatzbereiche des Fragmentes sind in der Posteingangsbearbeitung oder der Archivierung papiergebundener Dokumente zu finden (Mertins 2008, S. 80f). Unternehmen mit dem Anspruch der elektronischen Langzeitarchivierung sind gezwungen, den Prozess der elektronischen Archivierung innerhalb des Unternehmens abzubilden oder in ausgelagerter Form (z.B. Outsourcing) umsetzten zu lassen. Entlang dieser Vorgehensweise wurden insgesamt sieben Fragmente erstellt: Digitalisierung, Erfassung, Bearbeitung, Veröffentlichung, Wiederverwendung, Archivierung und Löschung.

\section{Tabelle 3: Gestaltungshinweise Fragment 1.1, Indikator Interoperabilität (Auszug)}

\begin{tabular}{|c|}
\hline $\begin{array}{l}\text { Informations- und Dokumentenaustausch } \\
\text { Dokumentenaustauschformate: Für den systemseitigen Austausch von Dokumenten sollten gängige Standards } \\
\text { verwendet werden (z.B. XML). Die eingesetzten Systeme sollten dies unterstützen. }\end{array}$ \\
\hline $\begin{array}{l}\text { Dokumentenformate: Es wird empfohlen, gängige Dokumentenformate für den Austausch von Dokumenten } \\
\text { zu verwenden, z.B. PDF. }\end{array}$ \\
\hline $\begin{array}{l}\text { Dokumentenformate: Zum Dokumentenaustausch zwischen organisatorischen Einheiten innerhalb des Un- } \\
\text { ternehmens sollten gängige unternehmensweit genutzte Formate eingesetzt werden (z.B. MS WORD, Open } \\
\text { Office) }\end{array}$ \\
\hline $\begin{array}{l}\text { Die Ablage der digitalisierten Dokumente sollte in einem gängigen speicherarmen Format (z.B. TIFF, PDF/A) } \\
\text { erfolgen können. }\end{array}$ \\
\hline $\begin{array}{l}\text { Für den Austausch von Informationen zwischen organisatorischen Einheiten wird die Nutzung von E-Mails } \\
\text { empfohlen. Bei nicht dokumentationswürdigen Sachverhalten sollte eine telefonische Verständigung ange- } \\
\text { strebt werden. }\end{array}$ \\
\hline Prozessschnittstellen \\
\hline $\begin{array}{l}\text { Ausgehend vom Gesamtprozess sollten einheitliche Übergabeformate zwischen Prozessen, Prozessbausteinen } \\
\text { und Prozessschritten (Aktivitäten) definiert werden. }\end{array}$ \\
\hline $\begin{array}{l}\text { Es werden klar definierte Schnittstellen am Anfang und Ende des gesamten Prozesses sowie zwischen Pro- } \\
\text { zessbausteine zum Vor- und Nachfolger empfohlen. }\end{array}$ \\
\hline $\begin{array}{l}\text { Da die Prozessschritte vor der Ablage des Dokuments variabel sein können muss die Prozessschnittstelle zum } \\
\text { Prozessschritt „Dokument ablegen“ eindeutig definiert werden. }\end{array}$ \\
\hline Modul- und Systemschnittstellen \\
\hline $\begin{array}{l}\text { Zur Kommunikation zwischen Systemen und Systemmodulen sollten Standards, wie z.B. XML, EDI eingehal- } \\
\text { ten werden. Das genutzte System sollte mit anderen Systemen verbunden werden können (z.B. über SOA, } \\
\text { Web Service, direkte Einbindung von anderen Systemen oder Einbindung des Systems in andere Systeme). }\end{array}$ \\
\hline Transfer von Prozesselementen \\
\hline $\begin{array}{l}\text { Prozessschritte eines Prozessbausteins sollten bei Bedarf ausgelassen, verschoben oder ergänzt werden kön- } \\
\text { nen. }\end{array}$ \\
\hline
\end{tabular}

\subsection{Erstellung von Kartenmustern}

Basierend auf den erarbeiteten Fragmenten wird nun die Entwicklung von Kartenmustern aufgezeigt, die im Sinne einer Referenzmodellbildung typische ECMProzessbausteine unter dem Aspekt der Wiederverwendung darstellen. Referenzmodelle stellen grundsätzlich Artefakte mit Empfehlungscharakter dar, die Modellierer bei der Konstruktion individueller Prozesse anleiten sollen (Becker und Delfmann 2008, S. 339). Im Bereich des ECM besteht hinsichtlich der Referenzmodellierung enormer Handlungsbedarf. Derzeit existieren nur Hinweise zur Prozessgestaltung im DOMEA ${ }^{\circledR}$-Organisationskonzept (teilweise auch im MoReq2- 
Standard) sowie in einigen Spezifikationen konkreter Anwendungssysteme. Die grundlegende Idee der Kartenmuster ist die Beschreibung eines ECMProzessschrittes in wandlungsfähiger Form. Die Gestaltung eines Prozessschrittes basiert dabei auf einem standardisierten Prozess, welcher einen typischen ECMProzess abbildet. Die Beschreibung eines Kartenmusters besteht aus einem initialen Kontext, der einen allgemeinen, wiederkehrenden Ablauf innerhalb der Dokumentenbearbeitung beschreibt, einem Vorschlag einer wandlungsfähigeren Gestaltung sowie der visualisierten Abbildung in Form einer Prozesskarte. Die entwickelten Kartenmuster sollen als Referenzprozesse im Sinne einer wandlungsfähigen Gestaltung von ECM-Prozessen eingesetzt werden können. Ein sinnvoller Einsatzbereich stellt hierbei die Potenzialanalyse im Rahmen der Aufdeckung von Prozessverbesserungen dar. Die Ableitung von Kartenmustern, die sich durch die Eigenschaft der Wandlungsfähigkeit auszeichnen, erfolgt entlang der Gestaltungsempfehlungen der erarbeiteten Fragmente. Ein Kartenmuster beschreibt hierbei einen bestimmten Prozessbaustein, der häufig innerhalb von ECM-Landschaften auftritt. Kartenmuster stellen neben der eigentlichen Prozessmodellierung Möglichkeiten für eine wandlungsfähige Gestaltung dar.

Die Erstellung der Kartenmuster beruht auf einer 3-phasigen Vorgehensweise:

- Auswahl des Prozesses

Die Entwicklung eines Kartenmusters beginnt mit der Auswahl des Prozesses, der als Kartenmuster im Sinne der Referenzmodellierung abgebildet werden soll. Hier$\mathrm{zu}$ werden die innerhalb einer Umfrage erhobenen typischen ECM-Prozesse (Eggert und Gronau 2006, S. 38ff) genutzt. Nach der Auswahl des ECM-Prozesses wird dieser mit Hilfe standardisierter Prozessvorgaben abgebildet. Der Prozess, dessen Ableitung im Folgenden aufgezeigt wird, ist die „Übergabe von Archivgut“.

- Prozessdarstellung

Die Prozessdarstellung, bestehend aus einer an der standardisierten DOMEASpezifikation zur Übergabe von Archivgut orientierenden Beschreibung (DOMEA 2005, S. 88) und einer Darstellung in Form einer Prozesskarte, dient als Basis für die Anwendung der Fragmente.

- Entwicklung des Kartenmusters

Zur Ableitung des Kartenmusters „Übergabe von Archivgut“, welche wandlungsfähige Gestaltungsaspekte berücksichtigt, erfolgt zunächst eine Zuordnung von Fragmenten. Der Prozesskarte „Übergabe von Archivgut“ wird das „Fragment 5.1: Archivierung" zugeordnet. Entlang des Fragments werden mögliche Potenziale aufgedeckt, die mit Hilfe der Gestaltungsempfehlungen konkretisiert werden. Die Ableitung wird in Abbildung 4 beispielhaft anhand der Gestaltungsempfehlungen der Indikatoren Interoperabilität und Skalierbarkeit visualisiert. 


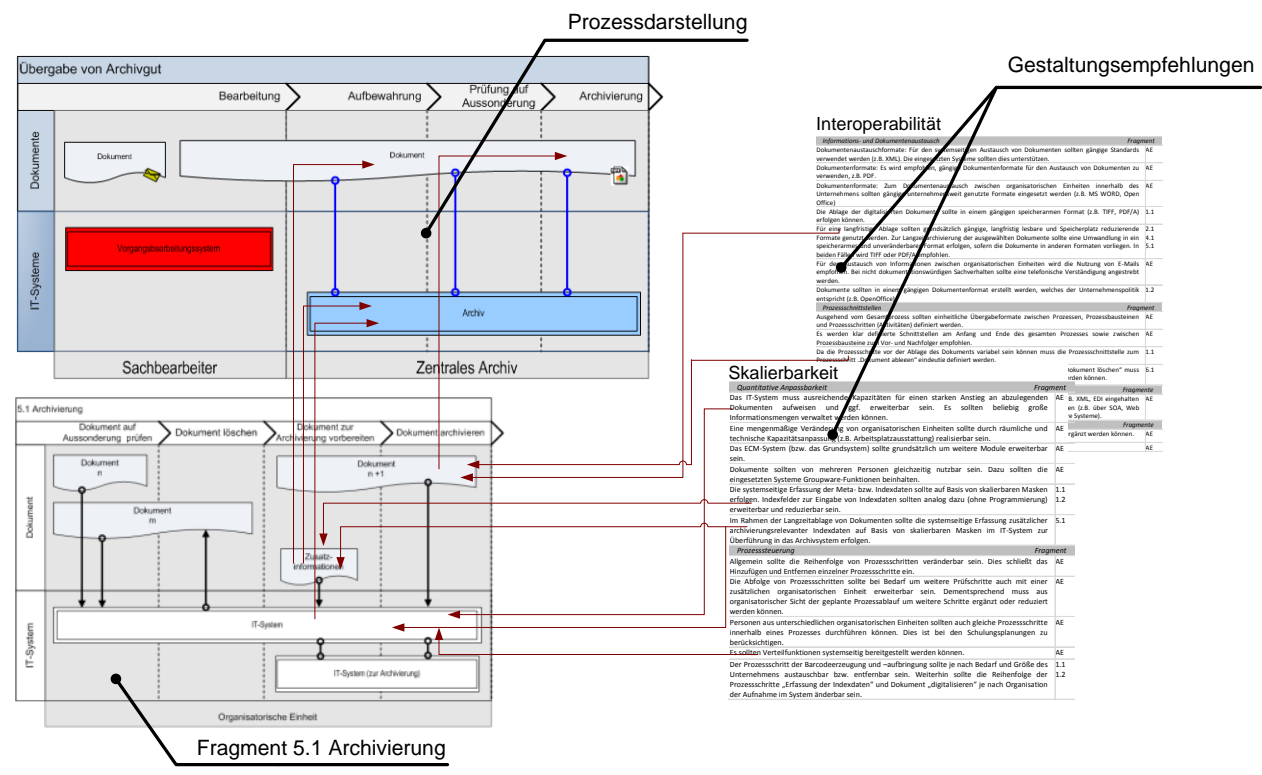

\section{Abbildung 4: Ausschnitt der Ableitung von Potenzialen}

Neben den in Abbildung 4 aufgezeigten Potenzialen wurden weitere Potenziale aufgrund der Gestaltungsempfehlungen entlang der weiteren Indikatoren identifiziert.

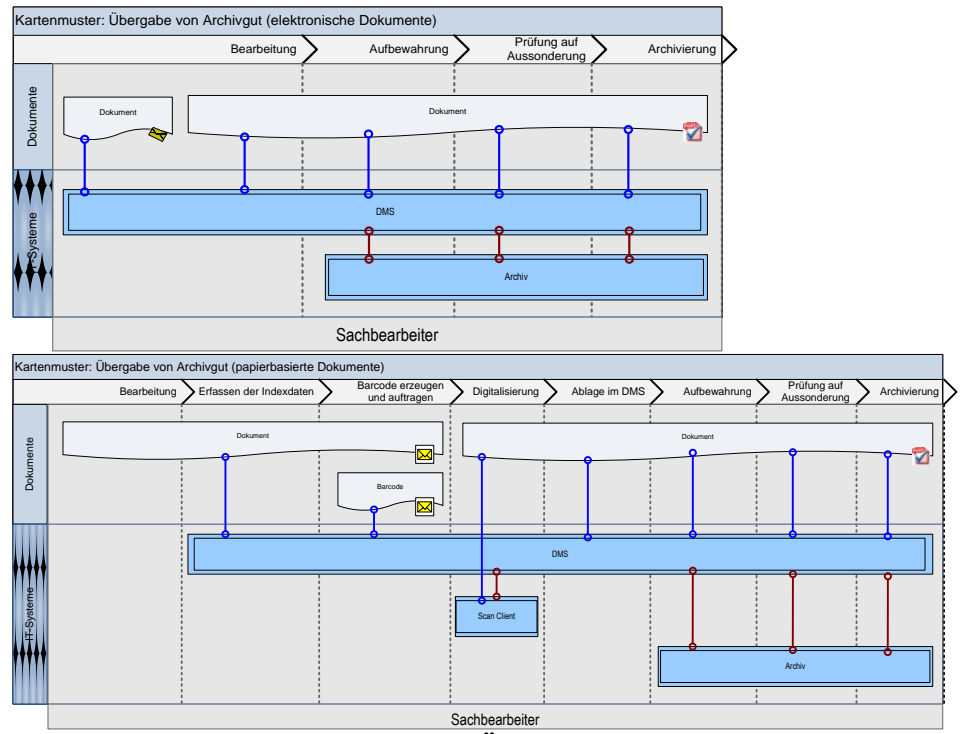

Abbildung 5: Kartenmuster zur Übergabe von elektron. und papierbas. Archivgut 
Die Umsetzung dieser Gestaltungsempfehlungen mündet aufgrund der Differenzierung in elektronische und papierbasierte Dokumente in die Kartenmuster zur „Übergabe von elektronischem Archivgut" und zur „Übergabe von papierbasiertem Archivgut“ (Darstellung in Abbildung 5).

\section{Anwendungsbeispiel einer Prozessanalyse}

Die auf Basis der Fragmente entwickelten Kartenmuster wurden innerhalb von zwei Fallstudien evaluiert. Dazu wurden Unternehmen ausgewählt, die sich in einem turbulenten Umfeld befinden und einen Bedarf an Prozessverbesserung im Bereich ihrer ECM-Prozesse identifiziert haben. Zunächst wurden die IST-Prozesse aufgenommen und visualisiert. Beispielhaft wird die Ableitung der Potenziale anhand des Prozesses „Einreichen der Drittmittelanzeige“ aufgezeigt. Für diesen Prozess werden die Kartenmuster „Interne Antragstellung“ und „Übergabe von Archivgut" verwendet. Abbildung 6 zeigt die Analyse der Potenziale.

Folgende Potenziale wurden identifiziert:

1. Die Dokumentenvorhaltung im Intranet sollte durch die Nutzung des ECM als unternehmensweites Portal mit personifiziertem Arbeitsbereich abgelöst werden.

2. Durch die Bearbeitung innerhalb von PDF-Dokumenten sollte eine medienbruchfreie Übertragung des PDF-Dokuments realisiert werden.

3. Durch die Vermeidung von Medienbrüchen entfällt der Schritt „Ausdrucken“.

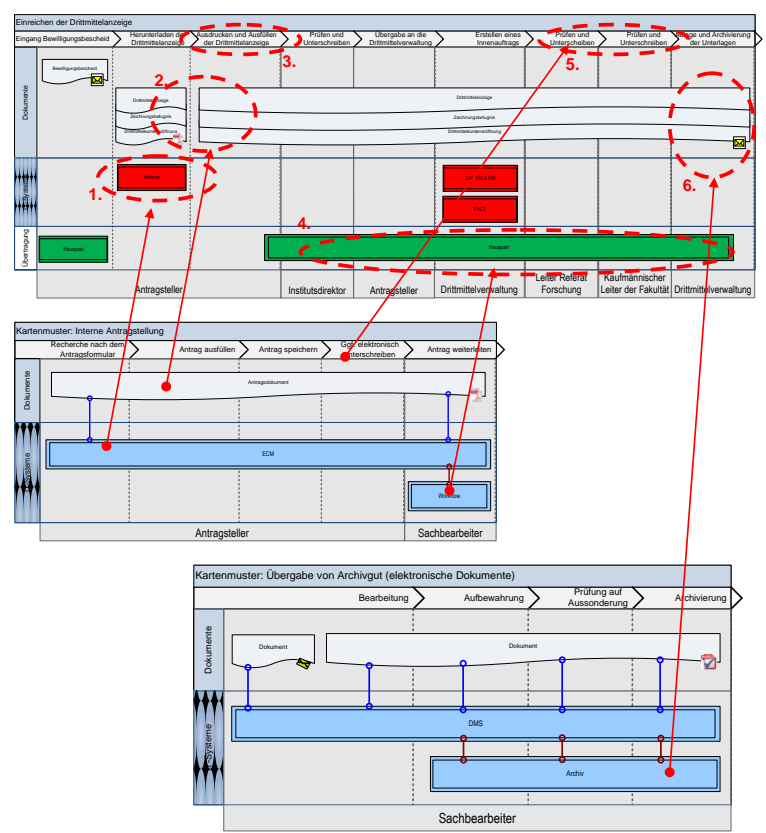

Abbildung 6: Potenzialanalyse - Prozess: Einrichten der Drittmittelanzeige 
4. Die Übertragung von Dokumenten erfolgt workflowgesteuert über das ECM-System. Der Übertragungsweg der Hauspost kann somit vermieden werden.

5. Das Unterzeichnen von Dokumenten kann mit Hilfe der elektronischen Signatur elektronisch im PDF-Dokument erfolgen. Dies verspricht eine Zeiteinsparung hinsichtlich der Vermeidung des Übertragungswegs der Hauspost.

6. Die Archivierung der Dokumente erfolgt elektronisch im Archiv-Modul des ECM.

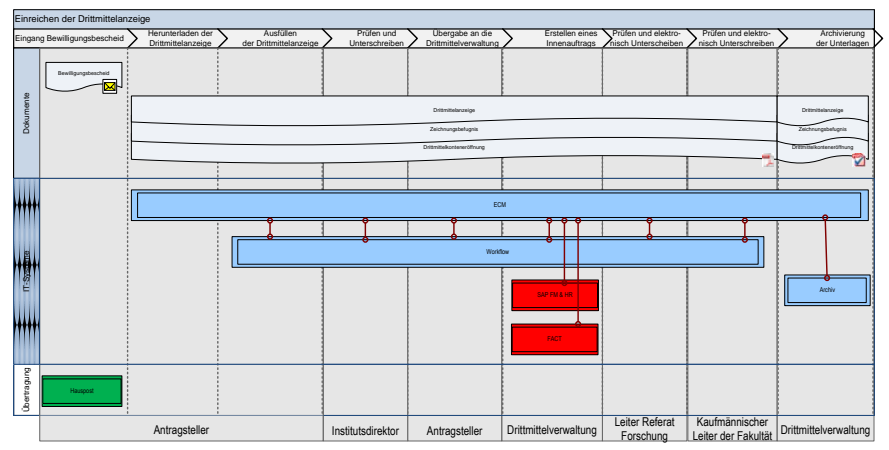

\section{Abbildung 7: SOLL-Prozess des Einreichens der Drittmittelanzeige}

Anhand der ermittelten Potenziale konnten SOLL-Prozesse abgeleitet werden. Ziel der Sollmodellierung ist es, alternative Szenarien für wandlungsfähigere Prozesse aufzuzeigen, die die identifizierten Potenziale mit Hilfe der wandlungsfähigen Kartenmuster beinhalten. Die Umsetzung der erarbeiteten SOLL-Prozesse wurde in beiden Unternehmen geplant. In einem Unternehmen hat die Umstellung der Prozesse bereits begonnen. Die direkte Gegenüberstellung von IST- und SOLLProzessen zur Prüfung auf Erhöhung der Wandlungsfähigkeit ist jedoch erst nach vollständiger Umsetzung aller geplanten Maßnahmen möglich. Infolgedessen kann eine Überprüfung derzeit nur anhand der abgeleiteten SOLL-Prozesse mittels der Indikatoren für Wandlungsfähigkeit durchgeführt werden.

Tabelle 4: Prüfung der SOLL-Prozesse hinsichtl. der Wandlungsfähigkeitssteigerung

\begin{tabular}{|l|l|c|}
\hline Indikatoren & Maßnahmen entlang der SOLL-Prozesse & $\begin{array}{l}\text { Bewertung } \\
\text { Wandlungsf. }\end{array}$ \\
\hline Modularität & Einsatz eines modular aufgebauten ECM-Systems & + \\
\hline Skalierbarkeit & $\begin{array}{l}\text { durch die Vermeidung der Hauspost können mehr Prozessschritte } \\
\text { in gleicher Zeit abgearbeitet warden }\end{array}$ & + \\
\hline Mobilität & $\begin{array}{l}\text { Vermeidung von Medienbrüchen, die Übertragung von Dokumen- } \\
\text { ten erfolgt elektronisch }\end{array}$ & ++ \\
\hline Redundanz & zentrale Ablage im ECM, Backuprepliken der ECM-Daten & + \\
\hline Selbstähnlichkeit & Verwendung gleicher elektronischer Formate & + \\
\hline Selbstorganisation & die Abhängigkeit von der Hauspost entfällt weitgehend & + \\
\hline Wissen & $\begin{array}{l}\text { Durch die zentrale Nutzung des ECM sind einer höheren Mitarbei- } \\
\text { teranzahl die Dokumente des Prozesses zugänglich (Prozesswis- } \\
\text { sen), Dokumentationen zu den ECM-Modulen sowie Hilfefunkti- } \\
\text { onen in den Systemen }\end{array}$ & + \\
\hline
\end{tabular}


Für den in diesem Beitrag beispielhaft abgeleiteten SOLL-Prozess „Einreichen der Drittmittelanzeige“ zeigt Tabelle 4 die Bewertung der Wandlungsfähigkeit.

\section{Zusammenfassung und Ausblick}

Die Bewertung der SOLL-Prozesse der beiden Fallstudien hinsichtlich einer Veränderung der Wandlungsfähigkeit weist summarisch eine deutliche Erhöhung gegenüber den IST-Prozessen auf. Dies zeigt, dass bei Umsetzung der SOLLProzesse eine höhere Wandlungsfähigkeit der Prozesse zu erwarten ist. Damit einher geht auch eine schnellere und flexiblere Reaktionsfähigkeit der betroffenen Unternehmensbereiche. Dies bestätigt einerseits die Praxistauglichkeit der Kartenmuster sowie den Einsatz der Kartenmuster innerhalb der Potenzialanalyse als sinnvoll. Der vorliegende Beitrag stellt in Hinblick auf eine wandlungsfähige Gestaltung von ECM-Prozessen einen Ansatz dar. Es wurde eine Vorgehensweise erarbeitet, die es ermöglicht, aus den entwickelten Fragmenten, Kartenmuster zu erstellen, die während einer Potenzialanalyse in der Praxis eingesetzt werden können.

\section{Literatur}

Andresen K, Levina O, Gronau N (2008) Design of the evolutionary process model for adaptable software development processes. In: Proceedings of the EMCIS

Becker J; Delfmann P (2008) Referenzmodellierung 2008. In: Proceedings of the MKWI. M. Bichler et al.: 339-340.

DOMEA ${ }^{\circledR}$-Konzept, Organisationskonzept 2.1, http://www.verwaltunginnovativ.de. Abruf am 2008-01-09

Eggert S, Gronau N (2005) Auswertung einer Marktstudie zu Enterprise Content Management Systemen. In: ERP Management, 4: 48-59.

Eggert S, Gronau N (2006) Anbieter überschätzen Wandlungsfähigkeit ihrer ECMLösungen. In: isreport 7+8: 38-41.

Eggert S, Gronau N (2008) Erhöhung der Wandlungsfähigkeit von ECM-

Lösungen unter Verwendung kartographischer Gestaltungsmittel. In:

Proceedings of the MKWI. M. Bichler et al.: 433-446.

Gronau N, Wildemann H, Zäh M F Müller N Lämmer A Andresen K (2006)

Tools zur Ermittlung der Wandlungsfähigkeit. In: ERP Management 1: 23-26.

Heinen T, Gerst D, Nyhuis P (2008) Soziotechnische Planung der

Wandlungsfähigkeit. Methodik zur Planung und Gestaltung der 
Wandlungsfähigkeit auf Basis von Geschäftsprozessen. In: Werkstattstechnik online, 98: 403-408.

Kaiser M G, Smolnik S, Riempp G (2008) Konzeption eines InformationLifecycle-Management-Frameworks im Dokumenten-Management-Kontext. In: Proceedings of the MKWI. M. Bichler et al.: 483-494.

Mertins K, Kohl H, Görmer M (2008) Benchmarking-Studie Dokumentenlogistik. Eine Unternehmensbefragung zum Stand der Dokumentenlogistik in Deutschland. Fraunhofer IPK Eigenverlag, Berlin.

Möller N, Rimpau C, Müssig B, Dietrich A (2005) Bewertung von Wandlungsfähigkeit im Rahmen der Fabrikplanung. In: iwb Newsletter, 13/2: 2-3.

Overby E, Bharadwaj A, Sambamurthy V (2006) Enterprise agility and the enabling role of information technology. In: European Journal of Information Systems, 15: 120-131.

Rogalski S, Ovtcharova J (2009) Flexibilitätsbewertung von Produktionssystemen, ecoFLEX - eine branchenübergreifende Methodik. In: ZWF Zeitschrift für wirtschaftlichen Fabrikbetrieb, 104/1-2: 64-70.

Wiendahl H.-P (2009) Veränderungsfähigkeit von Produktionsunternehmen. In: ZWF Zeitschrift für wirtschaftlichen Fabrikbetrieb, 104/ 1-2: 32-37.

Wildemann H (2006) Erhöhung der Wandlungsfähigkeit in der Auftragsabwicklung. In: Wandlungsfähige ERP-Systeme. Entwicklung, Auswahl und Methoden. Gronau N, Lämmer A (Hrsg): 1-17. 